\title{
Hydrodechlorination and Hydrogenation over Raney-Ni under Multiphase Conditions: Role of Multiphase Environment in Reaction Kinetics and Selectivity
}

\author{
Sergei Zinovyev, ${ }^{*}, \ddagger$ Alvise Perosa,$\dagger$ Sergei Yufit,$\ddagger$ and Pietro Tundo $\dagger, 1$ \\ *Interuniversity Consortium "Chemistry for the Environment” (INCA), Dorsoduro 2137, 30123 Venezia, Italy; †Ca' Foscari University of Venice, \\ Dorsoduro 2137, 30123 Venezia, Italy; and $\ddagger$ Zelinsky Institute of Organic Chemistry of Russian Academy \\ of Sciences, Leninsky Pr. 47, Moscow 119991, Russia
}

Received March 4, 2002; revised July 18, 2002; accepted July 18, 2002

The hydrodechlorination (HDC) and hydrogenation (HG) of 1,3,5-trichlorobenzene (TCB) and of $p$-chloroacetophenone with hydrogen at atmospheric pressure and at $50^{\circ} \mathrm{C}$ over Raney-Ni in a biphasic organic-aqueous system and in the presence of a phasetransfer agent (Aliquat 336) were studied from a kinetic standpoint. The nature and the concentration of the aqueous base, as well as the concentration of Aliquat 336, were investigated. HDC of $1,3,5-\mathrm{TCB}$ proceeds only in the presence of base and Aliquat 336 , and the dechlorination rate of each subsequent chlorine removal increases by one order of magnitude. The highest rates are observed at around $3 \% \mathrm{KOH}$ or $10 \% \mathrm{~K}_{2} \mathrm{CO}_{3}$. Carbonates and bicarbonates of potassium and sodium are shown to be better bases than $\mathrm{KOH}$. Concentrated aqueous $\mathrm{KOH}$ inhibits the reaction, especially if used without Aliquat 336. The dependence of dechlorination rates on the concentration of Aliquat 336 suggests that the phase-transfer agent is adsorbed on the catalytic surface. For $p$-chloroacetophenone the dechlorination step always precedes reduction of carbonyl, even if is slower that the latter. The roles of base and of Aliquat 336 are not critical in this case, although higher $\mathrm{KOH}$ concentrations enhance dechlorination and inhibit reduction of hydroxyl in 1-phenyl ethanol. (c) 2002 Elsevier Science (USA)

Key Words: Raney-nickel; hydrogenation; hydrodechlorination; multiphase conditions; kinetics.

\section{INTRODUCTION}

We have previously reported on a number of applications of the multiphase conditions for hydrodechlorination (HDC) and hydrogenation (HG) reactions of various polyhalo- (1-5) and other functional aromatics (5-8) over supported $\mathrm{Pt}(6-8)$ and $\mathrm{Pd}(1-5)$ catalysts. The multiphase system is made by a biphasic aqueous-organic medium, a heterogeneous catalyst, and a phase-transfer (PT) agent, and it operates at atmospheric pressure of hydrogen and

\footnotetext{
${ }^{1}$ To whom correspondence should be addressed. Fax: +39 (041) 234 8620. E-mail: tundop@unive.it.
}

moderate reaction temperatures $\left(50^{\circ} \mathrm{C}\right)$. The presence of a base in the aqueous phase is also critical, especially for the HDC reaction, and its presence can also steer selectivity in the reduction of other functional groups, such as carbonyl, hydroxyl, and aromatic ring (6-8).

The "multiphase environment" has a number of advantages over the traditional gaseous-phase $\mathrm{HDC} / \mathrm{HG}$ : (a) the mild reaction conditions (temperature and pressure) are easily accessible and generally afford a more selective and cleaner reaction with high yields; (b) catalyst poisoning/ deactivation by $\mathrm{HCl}(9)$ or other side products is negligible under such conditions; (c) the tunability of the system is such that by varying the composition of the reaction mixture one can direct the outcome selectively toward one of the possible reduction products. The PT agent (3-6), the $\mathrm{pH}$ of the aqueous phase $(7,8)$, and the ratio of the PT agent and heterogeneous catalyst (6) all have been found to be important factors.

The selectivity, kinetics, and synthetic $(10,11)$ and environmental applications (12) of the multiphase systems have been amply studied for charcoal-supported Pt and Pd catalysts. Some mechanistic hypotheses on the mode of action of the multiphase environment have been proposed as well (7). Raney-Ni, on the other hand, has been also found to be active under multiphase conditions in the hydrodehalogenation of polyhalobenzenes (3), but no detailed study has been performed on the role of the multiphase environment in its presence. The main advantages of Raney-Ni are its cost and availability, which compensate for its lower activity with respect to $\mathrm{Pd}$ or Pt.

The present study deals with the Raney-Ni-catalyzed $\mathrm{HDC} / \mathrm{HG}$ of 1,3,5-trichlorobenzene (TCB) and $p$-chloroacetophenone. The former is an interesting substrate in view of the possible environmental applications of the multiphase catalytic HDC for detoxification of hazardous polyhaloaromatics (12), and the latter is an example of a polyfunctional aromatic and is interesting from synthetic and mechanistic standpoints, since dechlorination versus 
reduction of carbonyl and aromatic rings may occur, bringing into play some selectivity features.

Particular attention is paid to the investigation of the roles played by the PT agent (Aliquat $336^{\circledR}$, tricaprylmethylammonium chloride) and the aqueous base, which are known to have a decisive role in the reaction selectivity (3-8). A kinetic study of these reactions allows measuring the rate constants for the independent steps, which provide a straightforward estimation of the selectivity of the reaction.

\section{METHODS}

All the reagents and solvents were ACS grade and were used without further purification. The Raney-Ni $(50 \%$ in water) was from Engelhard, Actimet $\mathrm{M}^{\mathrm{TM}}$, Lot H-482, composed of $93 \% \mathrm{Ni}$ and $7 \% \mathrm{Al}$, with a particle size distribution of $0-80 \mu \mathrm{m}$. The $\mathrm{KH}_{2} \mathrm{PO}_{4}-\mathrm{NaOH}$ buffer solution of $\mathrm{pH}$ 7.00 was from Carlo Erba. GC analyses were performed on a Varian 3400 using a fused silica capillary column "Chrompack CP-Sil 24 CB lowbleed/MS" $(30 \mathrm{~m} \times 0.25 \mathrm{~mm}$, film thickness $0.25 \mu \mathrm{m})$. GC/MS analyses were performed on a HP 5971 mass detector coupled to a HP 5890 gas chromatograph with a $30 \mathrm{~m} \times 0.25 \mathrm{~mm}$ DB5 capillary column.

\section{General Procedure for HDC of 1,3,5-TCB (Conditions of Entry 4, Table 1)}

A 25-ml three-necked round-bottomed flask thermostated at the reaction temperature $50 \pm 0.1^{\circ} \mathrm{C}$, connected with a system for the addition of hydrogen and with a waterjacketed condenser, was employed for the reaction. A 10-ml isooctane solution of Aliquat 336 (tricaprylmethylammonium chloride, $0.103 \mathrm{~g}, 0.26 \mathrm{mmol}$ ) and $n$-dodecane as the internal standard $(0.059 \mathrm{~g}, 0.35 \mathrm{mmol})$ and a $50 \%$ suspension of Raney-Ni in water $(0.25 \mathrm{~g}, 2 \mathrm{mmol}$ of $\mathrm{Ni})$ were placed in to the reactor. The hydrogen supply was turned on $(10 \mathrm{ml} / \mathrm{min}$ at atmospheric pressure $)$ and the mixture was stirred magnetically for 2-5 min, after which $5.7 \mathrm{ml}$ of $3 \%$ aqueous $\mathrm{KOH}$ was added $(3 \mathrm{mmol}$ of $\mathrm{KOH})$. After subsequent stirring of the mixture for nearly $5 \mathrm{~min}, 0.127 \mathrm{~g}$ of substrate $(0.7 \mathrm{mmol})$ was added, and this point was considered the beginning of the reaction (zero-time point at which GC sample was taken). The reaction was stirred magnetically at $1000 \mathrm{rpm}$ and bubbled with hydrogen.

\section{General Procedure for $\mathrm{HG}$ of p-Chloroacetophenone}

(Conditions of Entry 4, Table 3)

A 25-ml three-necked round-bottomed flask thermostated at the reaction temperature $50 \pm 0.1^{\circ} \mathrm{C}$, connected with a system for the addition of hydrogen and with a waterjacketed condenser, was employed for the reaction. A 10$\mathrm{ml}$ isooctane solution of Aliquat 336 (tricaprylmethylammonium chloride, $0.103 \mathrm{~g}, 0.26 \mathrm{mmol}$ ) and $n$-decane as the internal standard $(0.0407 \mathrm{~g}, 0.3 \mathrm{mmol})$ and a $50 \%$ suspension of Raney-Ni in water $(0.25 \mathrm{~g}, 2 \mathrm{mmol}$ of $\mathrm{Ni})$ were added to the reactor. The hydrogen supply was turned on $(10 \mathrm{ml} / \mathrm{min}$ at atmospheric pressure) and the mixture was stirred magnetically for $2-5 \mathrm{~min}$, after which $5.7 \mathrm{ml}$ of $1 \%$ aqueous $\mathrm{KOH}$ was added ( $1 \mathrm{mmol}$ of $\mathrm{KOH})$. After subsequent stirring of the mixture for nearly $5 \mathrm{~min} 0.086 \mathrm{ml}$ of substrate $(0.7 \mathrm{mmol})$ was added, and this point was considered the beginning of the reaction (zero-time point at which GC sample was taken). The reaction was stirred magnetically at $1000 \mathrm{rpm}$ and bubbled with hydrogen.

For all procedures the concentration of base in the aqueous phase was maintained constant with respect to the molar amount of aromatic chlorine $(3 \% \mathrm{KOH}$ for $1,3,5-$ TCB and $1 \% \mathrm{KOH}$ for $p$-chloroacetophenone). In the experiments with more concentrated solutions of base the volume of the aqueous phase was always kept constant, i.e., $5.7 \mathrm{ml}$. In all the experiments the overall volume of the organic phase was always kept constant, i.e., $10 \mathrm{ml}$.

\section{Sampling Procedure and GC Analyses}

The samples were collected from the organic phase during the reaction at time intervals. The first one was taken just after the reagents had been loaded and it was considered the zero-time point. About $20 \mu \mathrm{l}$ of the reaction mixture was taken for each sample, which was then diluted with ethyl ether up to 1-2 $\mathrm{ml}$. The resulting sample was then shaken with silica and filtered with cotton wool before injection.

The analysis of the reaction components was performed using the internal standard technique (8). A number of calibration coefficients were calculated for each reaction component by analyzing the binary mixtures of these compounds with $n$-decane or with $n$-dodecane. The calibration coefficients $\left(k_{\mathrm{cal}}\right)$ for $p$-chloroacetophenone, acetophenone, phenyl ethanol, and ethyl benzene were calculated as follows: $0.046,0.039,0.038$, and 0.026 (with decane). The calibration coefficients $\left(k_{\text {cal }}\right)$ for 1,3,5-trichlorobenzene, 1,3dichlorobenzene, chlorobenzene, and benzene were calculated as follows: $0.07,0.065,0.075$, and 0.076 (with dodecane). The following expression was used for the calculation of component concentrations: $C_{\text {comp }}=k_{\text {cal }}\left(S_{\text {comp }} / S_{\text {stand }}\right)$, where $S_{\text {comp }}$ and $S_{\text {stand }}$ are the GC peak area units for the analyzed compound and for the internal standard, respectively. The results were successfully tested for reproducibility. The systematic error for reproduction, reflected in the determination of the rate constants, was no higher than $20 \%$. The first-order rate constants were obtained by integral rate analysis, using a nonlinear fitting technique described elsewhere (8) with rate expressions [1]-[4]. The errors in the determination of the rate constants by nonlinear least-squares fitting for particular experiments are reported in the tables (" " in some cases indicates that the error was comparable or higher than the value of the respective constant). 


\section{RESULTS AND DISCUSSION}

\section{HDC of 1,3,5-Trichlorobenzene}

1,3,5-Trichlorobenzene (TCB) was chosen as the model substrate for the kinetic investigation of HDC of polychloroaromatics under multiphase conditions. Its reduction in the presence of Raney-Ni proceeds to give benzene according to the pathway of Scheme 1.

This model substrate has three chlorines in symmetrically equal positions, which makes the kinetic model very simple, affording first-order constants $\left(k_{1}, k_{2}\right.$, and $k_{3}$ according to Scheme 1).

Kinetic model and data processing. The kinetic model for this reaction has been obtained based on the $A \rightarrow B \rightarrow C \rightarrow D$ sequence of first order steps (13), assuming pseudo first order for all the reaction steps and assuming that the observed constants include also the adsorption components, according to the Langmuir-Hinshelwood approach, as explained elsewhere $(8,14)$. Contribution of adsorption terms, however, does not change the overall kinetic picture, and a comparison of the intrinsic rates is possible. In fact, the kinetic profiles obtained can be approximated using the first-order mathematical model.

For the $A \rightarrow B \rightarrow C \rightarrow D$ sequence of first-order steps the following integral rate expressions have been derived, starting from the respective differential rate equations and taking into account the mass balance:

$$
\begin{aligned}
{[A]=} & {[A]_{0} e^{-k_{1} t}, } \\
{[B]=} & {[A]_{0} \frac{k_{1}}{k_{2}-k_{1}}\left(e^{-k_{1} t}-e^{-k_{2} t}\right), } \\
{[C]=} & {[A]_{0} \frac{k_{1} k_{2}}{\left(k_{2}-k_{1}\right)\left(k_{3}-k_{1}\right)\left(k_{3}-k_{2}\right)}\left(\left(k_{3}-k_{2}\right) e^{-k_{1} t}\right.} \\
& \left.-\left(k_{3}-k_{1}\right) e^{-k_{2} t}+\left(k_{2}-k_{1}\right) e^{-k_{3} t}\right), \\
{[D]=} & {[A]_{0}\left(1-\frac{k_{2} k_{3}}{\left(k_{2}-k_{1}\right)\left(k_{3}-k_{1}\right)} e^{-k_{1} t}\right.} \\
& \left.+\frac{k_{1} k_{3}}{\left(k_{2}-k_{1}\right)\left(k_{3}-k_{2}\right)} e^{-k_{2} t}-\frac{k_{1} k_{2}}{\left(k_{3}-k_{1}\right)\left(k_{3}-k_{2}\right)} e^{-k_{3} t}\right) .
\end{aligned}
$$

Applying the multiple data-set fitting by means of the Origin ${ }^{\circledR}$ v. 6.0 PC software with this set of expressions one can obtain a set of computed parameters, i.e., three rate
TABLE 1

Effects of Aqueous Base and of PT Agent on Kinetics of HDC of 1,3,5-TCB over Raney-Ni under Multiphase Conditions

\begin{tabular}{llccccc}
\hline & \multicolumn{2}{c}{$\begin{array}{c}\text { Reaction mixture } \\
\text { composition }\end{array}$} & & \multicolumn{3}{c}{$\begin{array}{c}\text { First-order rate } \\
\text { constants }\left(\mathrm{min}^{-1}\right)\end{array}$} \\
\cline { 2 - 3 } \cline { 5 - 7 } No. & PTC & Base & & $k_{1}\left(\times 10^{3}\right)$ & $k_{2}\left(\times 10^{2}\right)$ & $k_{3}$ \\
\hline 1 & No & No, water & & 0 & - & - \\
2 & Yes & No, water & & 0 & - & - \\
3 & No & $3 \% \mathrm{KOH}$ & & 0 & - & - \\
4 & Yes & $3 \% \mathrm{KOH}$ & & $4.9 \pm 0.2$ & $3.4 \pm 0.4$ & $\sim 0.38$ \\
$5^{a}$ & Yes & $3 \% \mathrm{KOH}$ & & $36 \pm 5.0$ & $9.8 \pm 2.0$ & $\sim 0.24$ \\
\hline
\end{tabular}

Note. For conditions see Methods.

${ }^{a} \mathrm{Pd} / \mathrm{C}(5 \%)$ as the catalyst $(0.0455 \mathrm{~g}, 0.021 \mathrm{mmol}$ of $\mathrm{Pd})$.

constants in our case, from a single procedure and from a single experiment. A typical kinetic procedure has been explained in detail elsewhere (8). Kinetic constants calculated by this model are presented in the tables and figures. For determination of the rate constants most experiments were performed once, though the reproducibility of the data was initially ascertained (within 10\% error for determination of constants in most cases).

Role of multiphase environment. The presence of the PT agent (A336) and of the base in the aqueous phase both have an effect on kinetics. The results reported in Table 1 show that both PT agent and aqueous base play important roles in HDC; in the absence of either, or both, the reaction does not proceed at all (entries 1-3 of Table 1).

Based on visual observations we can also propose that the presence of water as well as of A336 seems to be favorable for the reaction since much better dispersion of the catalyst particles is attained. The PT agent in this case may play a role similar to that of a surfactant. If the metal catalyst (Raney-Ni) is introduced alone into the reaction, no dispersion is observed, i.e., both aqueous and organic phases stay clear and the catalyst sticks to the stirring bar and the reactor walls. Addition of A336 induces a good dispersion of catalyst in the organic phase, but for several minutes only, after which it is again aggregated and sticks to the walls. Only with addition of the aqueous phase is good and stable catalyst dispersion attained. In such a system the catalyst resides preferably at the phase boundary. Possibly, hydration of the catalyst on the one hand and its lipophilization by A336 on the other hand are important for its presence at the liquid-liquid phase boundary, where it holds access

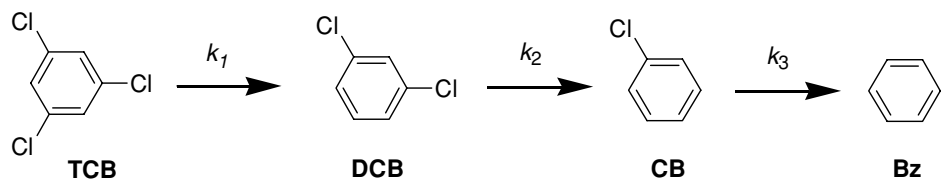

SCHEME 1. Raney-Ni, isooctane, aqueous $\mathrm{KOH}$, Aliquat $336^{\circledR}, 50^{\circ} \mathrm{C}, \mathrm{H}_{2}$ atmospheric pressure at $10 \mathrm{ml} / \mathrm{min}$. 
to both aqueous and organic phases and is more accessible by both the reacting substrate and the aqueous base.

In a typical multiphase system, i.e., with both PT agent and aqueous base (entry 4), the reaction proceeds selectively and with a high rate to the final yield of benzene. The dechlorination rates increase for the removal of each subsequent chlorine, i.e., the reactivity of chlorinated benzenes grows in the order TCB $<\mathrm{DCB}<\mathrm{CB}$. The same reactivity is observed in HDC of TCB over the supported Pd on carbon under the same conditions (entry 5). This behavior results in a virtual rate limitation by the first, slower step and does not make it possible to obtain high yields of intermediates (DCB and CB).

The results reported in Table 1 suggest that in the particular case of TCB dechlorination, A336 does not simply act as a PT catalyst and $\mathrm{KOH}$ does not simply neutralize $\mathrm{HCl}$ formed. In addition, the aqueous base and the PT agent seem to form a different environment on the catalytic surface or, possibly, to modify substrate coordination on the active site. Dechlorination over Raney-Ni in this system is a unique example, where the presence of these components is so critical. The previously reported results on dehalogenation of polyhalobenzenes over Raney-Ni have shown that also with dichloro- and dibromobenzenes $(o-, m-, p-)$, no reaction occurs in the absence of A336 (3).

Effect of the base. In order to gain a deeper insight into the role(s) of the PT agent and the base, the effect of $\mathrm{KOH}$ concentration in the aqueous phase on the reaction kinetics was investigated. The dependences of the calculated rate constants $k_{1}-k_{3}$ are presented in Fig. 1.

All the three constants seem to be affected in a similar manner by the basicity of the aqueous phase and show interesting correlations; that is, the highest rate is attained at

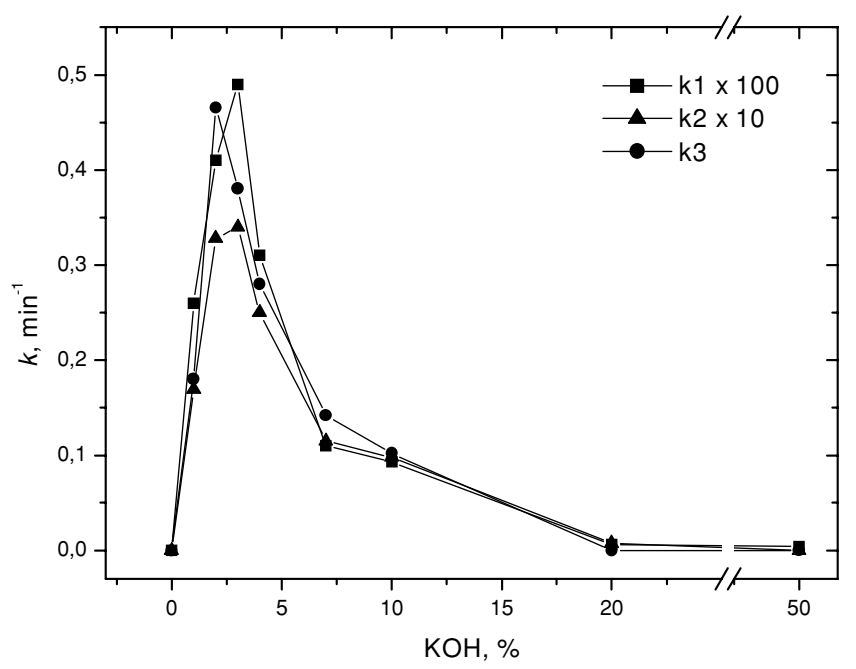

FIG. 1. Effect of aqueous $\mathrm{KOH}$ concentration on the dechlorination rate constants in HDC of 1,3,5-TCB over Raney-Ni under multiphase conditions (for conditions see Methods).

slightly basic aqueous solutions (approximately 3-4\%) and then sharply decreases with a further increase in base concentration. In fact, the reaction is very slow at $20 \%$ of base and is strongly inhibited at $50 \%$.

Rate inhibition by concentrated $\mathrm{KOH}$ is not clear and may be connected to the role of $\mathrm{KOH}$ as a strong dehydration agent (15) or to possible catalyst deactivation by $\mathrm{KOH}$, e.g., by hydroxylation of the residual Al (Raney-Ni used had $7 \%$ Al content).

A number of experiments were performed (Table 2) to explore the behavior of alternative bases, including the solid bases, which are known to be active in PT-catalyzed systems $(16,17)$. It was found that aqueous solutions of $\mathrm{KHCO}_{3}$,

TABLE 2

Alternative Base Component in Multiphase Catalytic HDC of 1,3,5-TCB over Raney-Ni

\begin{tabular}{|c|c|c|c|c|c|c|}
\hline \multirow[b]{2}{*}{ No. } & \multicolumn{3}{|c|}{ Reaction mixture composition } & \multicolumn{3}{|c|}{ First-order rate constants $\left(\min ^{-1}\right)$} \\
\hline & PTC & Base $^{a}$ & Solvent & $k_{1}\left(\times 10^{3}\right)$ & $k_{2}\left(\times 10^{2}\right)$ & $k_{3}$ \\
\hline 1 & No & $\mathrm{K}_{2} \mathrm{CO}_{3}$ solid & $96 \% \mathrm{EtOH}$ & $-^{b}$ & 0 & - \\
\hline 2 & Yes & $\mathrm{K}_{2} \mathrm{CO}_{3}$ solid & $96 \% \mathrm{EtOH}$ & 0 & - & - \\
\hline 3 & Yes & $\mathrm{K}_{2} \mathrm{CO}_{3}$ solid & Toluene & $-^{b}$ & 0 & - \\
\hline 4 & Yes & $\mathrm{K}_{2} \mathrm{CO}_{3}$ solid & Isooctane & $2.4 \pm 0.4^{c}$ & $1.1 \pm 1.1^{c}$ & $\sim 0.068^{c}$ \\
\hline 5 & Yes & $53 \% \mathrm{~K}_{2} \mathrm{CO}_{3}$ & Isooctane & $0.7 \pm 0.06$ & $0.69 \pm 0.45$ & $\sim 0.081$ \\
\hline 6 & Yes & $7.4 \%$ aq. $\mathrm{K}_{2} \mathrm{CO}_{3}$ & Isooctane & $15 \pm 0.3$ & $6.9 \pm 0.4$ & $0.65 \pm 0.34$ \\
\hline 7 & Yes & $\mathrm{NaHCO}_{3}$ solid & Isooctane & $0.52 \pm 0.06$ & $0.41 \pm 0.15$ & $\sim 0.039$ \\
\hline 8 & $\mathrm{No} / \mathrm{Yes}^{d}$ & $\mathrm{NaHCO}_{3}$ solid & EtOH abs. & $2.2 \pm 0.06$ & $1.3 \pm 0.06$ & $0.25 \pm 0.12$ \\
\hline 9 & Yes & $4.5 \%$ aq. $\mathrm{NaHCO}_{3}$ & Isooctane & $8.9 \pm 0.4$ & $4.9 \pm 2.1$ & $\sim 0.59$ \\
\hline 10 & Yes & $5.4 \%$ aq. $\mathrm{KHCO}_{3}$ & Isooctane & $12 \pm 0.3$ & $6.8 \pm 1.7$ & $0.59 \pm 0.45$ \\
\hline
\end{tabular}

Note. For conditions see Methods.

${ }^{a}$ In case of solid bases, tenfold molar excess of salt was used.

${ }^{b}$ Very low, could not be accurately determined.

${ }^{c}$ Rate constants were determined in the initial reaction period ( $10 \%$ conversion), after which the overall rate decreased in order of magnitude. With subsequent addition of water the rate increased again.

${ }^{d}$ A336 was added during the reaction course, but no pronounced change in kinetics was observed. 
$\mathrm{NaHCO}_{3}$, and $\mathrm{K}_{2} \mathrm{CO}_{3}$ all demonstrate apparently equal dechlorination rates on 1,3,5-TCB (entries 6, 9, 10, Table 2) and perform much better than $\mathrm{KOH}$. The reactions with different bases were carried out with the same amount of base equivalent with respect to chlorine as in the experiment with $3 \% \mathrm{KOH}$ (see entry 4 , Table 1 ). Sodium bicarbonate (entry 9) shows slightly slower reaction rates compared to the potassium salts.

Solid potassium carbonate was explored (Table 2, entries 1-4) but found to be less effective than the corresponding aqueous solution. Either no or a very slow reaction is observed (entries 1-4). In the presence of A336 and isooctane as solvent (entry 4) some reaction occurs in the very beginning, but then it becomes strongly suppressed. Then, if some amount of water is added to the reaction mixture the rate seems to recover to some extent. In fact, the reaction proceeds in the presence of a saturated solution of $\mathrm{K}_{2} \mathrm{CO}_{3}$ (entry 5), though much more slowly than in a moderately concentrated one (entry 6). When toluene is used instead of isooctane (entry 3 ) no conversion at all is observed, and in the presence of ethanol no reaction occurs with or without A336 (entries 1 and 2). Seemingly, in our system a PT agent cannot promote a sufficient transfer of anions from the solid salt surface to the catalytic surface of Raney-Ni, and the presence of water (present on Ni surface or coordinated to A336) is critical.

Contrary to potassium carbonate, the use of solid sodium bicarbonate with A336 in isooctane (entry 7) results in some, although quite slow, reaction. The rate is much higher when ethanol is used as solvent (entry 8 , Table 2), which is known to partially dissolve bicarbonates, making the direct transfer of anion to the catalytic surface possible. In this system (entry 8), the PT agent has no effect on reaction kinetics. This is an example of a classical system for the Raney-Ni-catalyzed reactions. However, it is less efficient than the multiphase one, since the rates are lower compared to the analogous systems with $\mathrm{K}_{2} \mathrm{CO}_{3}$ or $\mathrm{KHCO}_{3}$ and A336.

Since aqueous $\mathrm{K}_{2} \mathrm{CO}_{3}$ seems to be the base of choice in this system, we have studied the influence of $\mathrm{K}_{2} \mathrm{CO}_{3}$ concentration on the dechlorination rates (Fig. 2). Similarly to $\mathrm{KOH}$ (Fig. 1), the dependences of rate constants manifest in the optimal range of $\mathrm{K}_{2} \mathrm{CO}_{3}$ concentrations between 10 and $20 \%$. However, the observed range of "good" concentrations is wider than in the case of $\mathrm{KOH}$ and the rates do not decrease dramatically at higher concentrations. This easier trend of the rate constants using $\mathrm{K}_{2} \mathrm{CO}_{3}$ may be due to a number of reasons. Highly concentrated $\mathrm{K}_{2} \mathrm{CO}_{3}$ solutions are not as alkaline as $\mathrm{KOH}$ and do not retain water so intensively, causing dehydration of the organic phase as concentrated $\mathrm{KOH}(15)$. Generally speaking, the mechanistic role of the base in this system is unclear, but it is very important and seems to be more than just neutralization of $\mathrm{HCl}$.

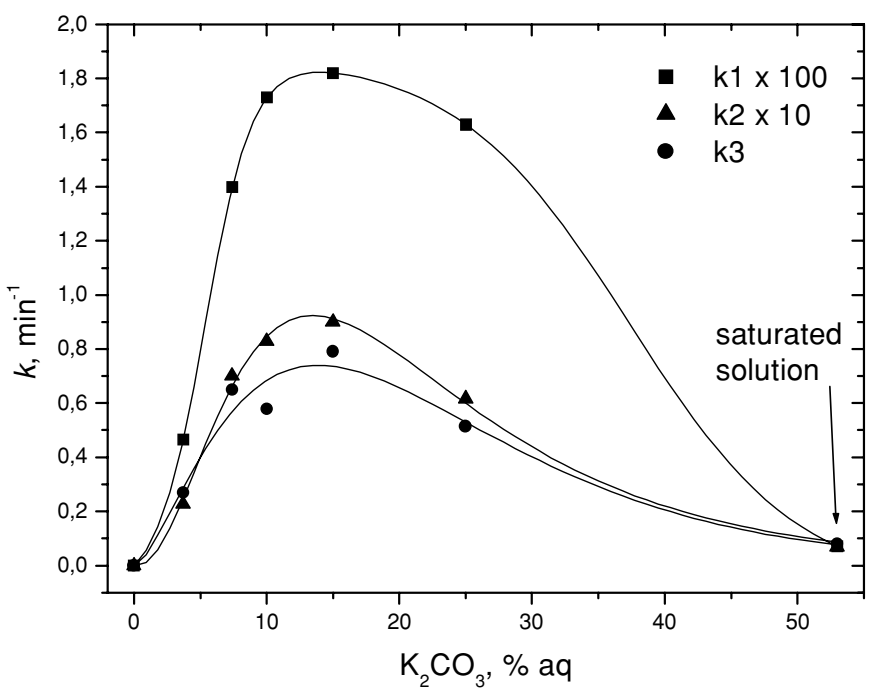

FIG. 2. Effect of $\mathrm{K}_{2} \mathrm{CO}_{3}$ concentration in HDC of 1,3,5-TCB over Raney-Ni under multiphase conditions (for conditions see Methods). Values of rate constants plotted at 7.4 and $53 \%$ refer to entries 6 and 5 of Table 2.

Effect of the PT agent. To investigate the effect of A336, the rates of 1,3,5-TCB HDC versus the A336 concentration were studied and are reported in Fig. 3.

All the dechlorination rates $\left(k_{1}, k_{2}, k_{3}\right)$ show similar trends with an increase in A336 concentration. Good linear dependences of the rate constants are observed at lower amounts of A336, indicating that the reaction is first order with respect to the PT agent at low concentrations of A336. However, the dependences show a sharp bend around $0.2 \mathrm{mmol}$ of A336, after which a further increase in the amount of A336 does not affect the rate constants.

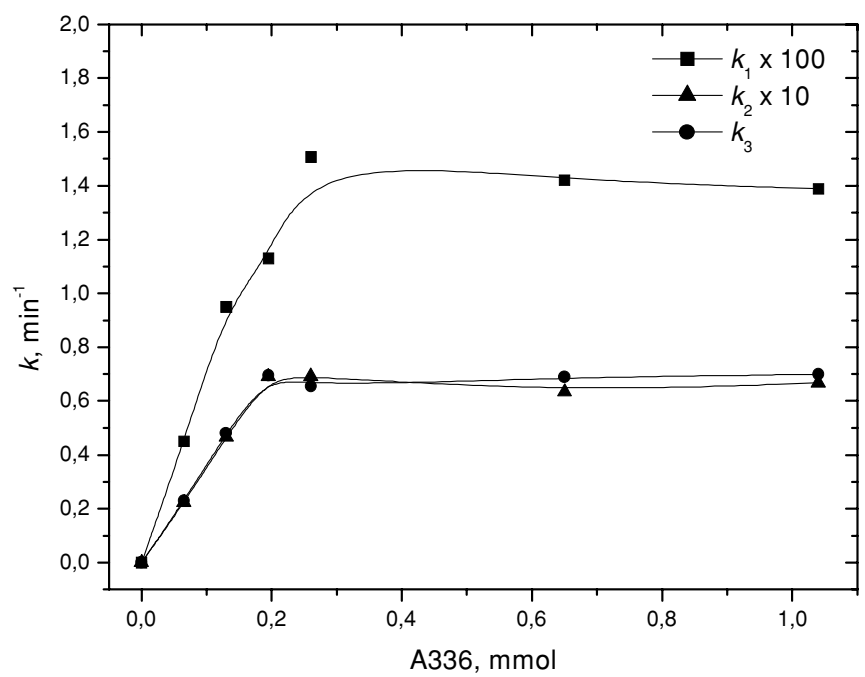

FIG. 3. Effect of Aliquat 336 concentration in HDC of 1,3,5-TCB over Raney-Ni with aqueous $7.4 \% \mathrm{~K}_{2} \mathrm{CO}_{3}$ (for conditions see Methods). 


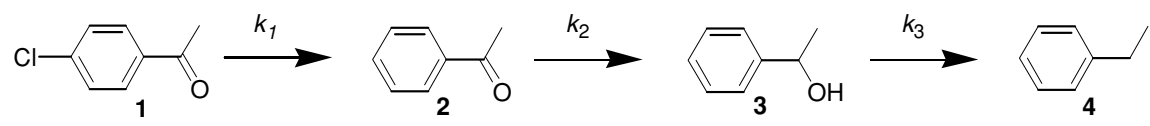

SCHEME 2

The variable order of the reaction in A336 suggests that the latter participates in the rate-determining step of the reaction, but its co-catalytic effect is produced by only those molecules of A336 that hold the direct access to the surface of the catalyst. Therefore, upon an exceeding amount of A336, i.e. when the surface is already saturated, it has no longer an influence over the reaction rates.

In fact, the dependences observed resemble the Langmuir-type adsorption curves, where the sharp bend of the curves is indicative of a stronger adsorption of the first than of the successive molecules. However, the amount of A336 $(0.2 \mathrm{mmol})$ at which the plateau of rate dependences is reached evidently exceeds the largest amount of A336 sufficient to form a monolayer in this system. A molecule of A336, arranged as compactly as possible, would cover an area with a radius of around three to four $\mathrm{C}-\mathrm{C}$ bonds, i.e., nearly $100 \AA^{2}$. For monolayer adsorption, $0.2 \mathrm{mmol}$ would cover $1.2 \times 10^{22} \AA^{2}$, or $1.2 \times 10^{2} \mathrm{~m}^{2}$. Since we have $0.125 \mathrm{~g}$ of Raney-Ni in our system, its calculated surface area would be around $10^{3} \mathrm{~m}^{2} / \mathrm{g}$, which is more than one order of magnitude higher than that reported by the manufacturer $\left(70 \mathrm{~m}^{2} / \mathrm{g}\right)(18)$. These preliminary considerations suggest that the adsorption of A336 over Raney-Ni either is not very strong or is competitive with other processes involving A336.

Remarkably, the dependences on the amount of A336 obtained for the reaction rates under study (Fig. 3) are the same as in the previously studied Pd/C-catalyzed hydrodehalogenation under similar conditions (4), where the saturation of the corresponding dependences also occurred at this amount of A336 and the ratio between the A336 amount and the $\mathrm{Pd} / \mathrm{C}$ surface area was also close to the present case.

The results discussed above do not build a direct link to an understanding of the true mechanistic role of the PT agent in our system. A more profound investigation into both the reaction kinetics and the adsorption of the PT agent, including comparison between different quaternary salts, is required. This is going to be a subject of our future investigations. Other experiments presented in the present work were carried out using $0.26 \mathrm{mmol}$ of A336, which is over the point of rate saturation in Fig. 3.

\section{HG and HDC of p-Chloroacetophenone}

The reductions of $p$-chloroacetophenone (8) and of other haloarylketones (5-7) have been previously studied over Pt- and Pd-supported on carbon catalysts under multiphase conditions. As a model substrate, $p$-chloroacetophenone can undergo different types of reductions: (i) dechlorination and (ii) reduction of carbonyl, (iii) hydroxyl, and (iv) aromatic rings. Previously, on acetophenone and $p$ chloroacetophenone the multiphase conditions were shown to be a very useful tool for tuning the reaction selectivity and for choosing among the possible reduction pathways by varying the composition of the reaction mixture (8). The present study is aimed at investigation of the behavior of Raney-Ni and, in particular, at understanding the roles played by the components of the multiphase environment, namely A336 and aqueous base. As in the case of 1,3,5-TCB, this study is approached from a kinetic standpoint, i.e., the rate constants are reported and discussed.

Generally, reduction of $p$-chloroacetophenone over Raney-Ni under multiphase conditions (with or without A336 and with water or slightly basic aqueous phase) proceeds toward ethyl benzene, as shown in Scheme 2. The same pathway was previously observed in the reduction of $p$-chloroacetophenone over $\mathrm{Pd} / \mathrm{C}$ in the absence of the PT agent (5), and it has been shown that the presence of a PT agent is the factor that changes the reaction selectivity from 4 (ethyl benzene) to $\mathbf{2}$ (acetophenone); that is, in the presence of A336 no reduction of carbonyl occurs. The reduction of chloroarylketones over $\mathrm{Pt} / \mathrm{C}(6,8)$ has been shown to proceed toward phenyl alcohols ( 3 in case of $p$-chloroacetophenone) in the presence A336 or toward cyclohexyl derivatives without A336 (6). The Raney-Nicatalyzed system also makes possible the tuning of this kind of selectivity, though in this case it depends on the aqueous base rather than on A336.

Since the pathway of Scheme 2 is kinetically identical to that of Scheme 1, the same kinetic model has been applied in this case; i.e., Eqs. [1]-[4] were used for calculation of the observed first-order rate constants $k_{1}-k_{3}$ (Scheme 2). The pseudo-first-order reaction was observed in most cases and all the data have been fitted with Eqs. [1]-[4].

In Table 3, the effects of the PT agent and of the aqueous $\mathrm{KOH}$ on the reaction kinetics are reported. It should be noted that contrary to dechlorinations of 1,3,5TCB and of dichlorobenzenes (3) dechlorination of $p$ chloroacetophenone proceeds in the absence of either A336 (entry 3) or base in the aqueous phase (entry 2), even somewhat more efficiently if neither base nor A336 is present (entry 1). Reduction of carbonyl in $\mathbf{2}$ (acetophenone) seems to not be affected by the presence of $\mathrm{KOH}$ and A336. Reduction of the hydroxyl group in $\mathbf{3}$ is favored in the absence of both (entry 1 in Table 3 ), which is similar to the behavior observed in the Pt-catalyzed reaction $(6,8)$, though, as is shown below, the presence of aqueous base 


\section{TABLE 3}

Effects of A336 and of Aqueous $\mathrm{KOH}$ on the Reaction Kinetics in HDC of $p$-Chloroacetophenone over Raney-Ni under Multiphase Conditions

\begin{tabular}{llllccc}
\hline & \multicolumn{2}{c}{$\begin{array}{c}\text { Reaction mixture } \\
\text { composition }\end{array}$} & & \multicolumn{3}{c}{$\begin{array}{c}\text { First-order rate } \\
\text { constants }\left(\mathrm{min}^{-1}\right)\end{array}$} \\
\cline { 2 - 3 } \cline { 5 - 6 } No. & A336 & Aqueous & & $k_{1}\left(\times 10^{3}\right)$ & $k_{2}\left(\times 10^{2}\right)$ & $k_{3}\left(\times 10^{4}\right)$ \\
\hline 1 & No & Water & & $2.8 \pm 0.16$ & $16 \pm 4$ & $39 \pm 4$ \\
2 & Yes & Water & & $1.5 \pm 0.06$ & $17 \pm 3$ & $22 \pm 2$ \\
3 & No & $3 \% \mathrm{KOH}$ & & $1.5 \pm 0.06$ & $20 \pm 9$ & $\sim 1$ \\
4 & Yes & $1 \% \mathrm{KOH}$ & & $3.5 \pm 0.4$ & $13 \pm 4$ & $16 \pm 4$ \\
5 & No & $50 \% \mathrm{KOH}$ & & 0 & - & - \\
6 & Yes & $50 \% \mathrm{KOH}$ & $-^{a}$ & $-^{a}$ & $-^{a}$ \\
$7^{b}$ & Yes $^{c}$ & $30 \% \mathrm{KOH}$ & & $20.1 \pm 1.8$ & $3 \pm 0.3$ & $\sim 1$ \\
8 & Yes $^{c}$ & $30 \% \mathrm{KOH}$ & & $12.0 \pm 1.1$ & $2 \pm 0.2$ & 0 \\
\hline
\end{tabular}

Note. For conditions see Methods.

${ }^{a}$ Very low, could not be accurately determined.

${ }^{b}$ Twofold excess of Raney-Ni.

${ }^{c}$ Threefold excess of A336.

seems to be more critical for reduction of hydroxyl. The conditions of entry 1 appear to be the best for the efficient reaction toward $\mathbf{4}$ since reduction of hydroxyl is always the rate-limiting step.

Effect of the base. The situation changes if a broader range of $\mathrm{KOH}$ concentrations is considered. In Fig. 4, rate constants $k_{1}-k_{3}$ are plotted against the $\mathrm{KOH}$ concentration, which varies from zero to $40 \%$.

As shown in Fig. 4, the dechlorination constant increases with an increase in $\mathrm{KOH}$ concentration, though this dependence is not linear and becomes less pronounced

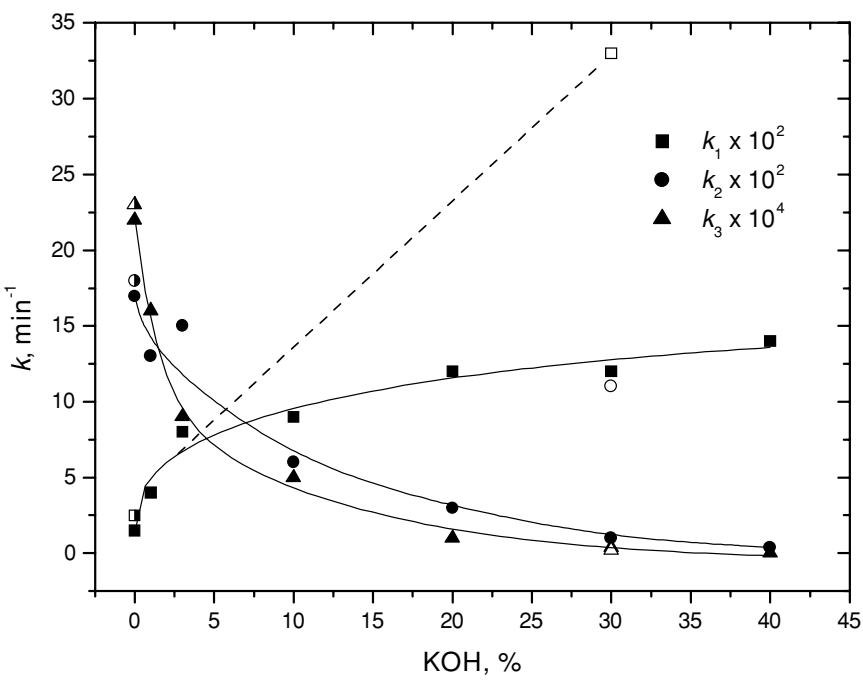

FIG. 4. Effect of aqueous $\mathrm{KOH}$ concentration on the rate constants of p-chloroacetophenone reduction over Raney-Ni under multiphase conditions with A336. (Half-solid symbols) The use of $\mathrm{pH} 7$ buffer as an aqueous phase; (open symbols) the experiment with more vigorous agitation of the reaction mixture. Rate constants reported at zero and $1 \% \mathrm{KOH}$ refer to entries 2 and 4 of Table 3 . with higher $\mathrm{KOH}$ concentrations. The constants of carbonyl and hydroxyl reductions, $k_{2}$ and $k_{3}$, respectively, instead decrease with higher $\mathrm{KOH}$ concentrations. The rate constant $k_{3}$ falls to zero at $40 \% \mathrm{KOH}$, and at $20 \%$ it is already so low that the reaction gives $\mathbf{3}$ selectively. The base is favorable for removal of chlorine, especially at higher concentrations, where the dechlorination rate is higher than that of carbonyl reduction; however, the latter is faster in the presence of neutral aqueous phase. The experiment with neutral buffer solution (Fig. 4) does not show much difference from that with simple water. Seemingly, the small amount of $\mathrm{HCl}$ formed during HDC either does not affect the reaction or reacts immediately with $\mathrm{Ni}$ (taken in threefold excess to the substrate).

More vigorous agitation of the reaction with highly concentrated $\mathrm{KOH}(30 \%)$, attained by using a different shape of stirring bar, results in a substantial increase in reaction rates (Fig. 4). This result is quite unexpected, because the range of stirring speeds used (approximately 900-1100 rpm) corresponds usually to the "kinetic region" in most biphasic systems, implying that higher stirring speeds should not affect the rates. Nevertheless, it must be noted that concentrated $\mathrm{KOH}$ is considerably dense (around $1.4 \mathrm{~g} / \mathrm{ml}$ for $50 \%$ $\mathrm{KOH})$ and viscous, which causes formation of emulsions in the biphasic systems, so the role of stirring is critical (16). Visually, a poorer phase dispersion has been observed under these conditions. The imaginary tendency presented as a dotted line in Fig. 4 depicts a hypothetical linear dependence of dechlorination rate on the $\mathrm{KOH}$ concentration, i.e., if the mass-transfer contribution arising from poor stirring could be eliminated.

It was noticed that aqueous $\mathrm{KOH}$ sometimes has a strong inhibition effect when put in contact with Raney-Ni before the PT agent is loaded into the system. In connection with this, the order of loading the reagents was found to be very significant (see Methods). Possibly, the PT agent, having been absorbed on the catalyst surface, preserves the latter from the deactivation by concentrated base and, therefore, has to be put in contact with Raney Ni several minutes before the base is added. With high concentrations of $\mathrm{KOH}$ full prohibition of the reaction occurs, as reported in entries 5 and 6 of Table 3 . These two results refer to a single experiment, which was run with the concentrated base and without A336 (entry 5) for $2 \mathrm{~h}$, and after it was noticed that no conversion occurred, the PT agent was added (entry 6). However, upon adding A336 the reaction did not start, contrary to the tendencies shown in Fig. 4, where quite good reaction occurred in the presence of $40 \% \mathrm{KOH}$. Less-concentrated $\mathrm{KOH}$ seemed not to cause such a pronounced inhibition (see entry 3 ), but even in this case $k_{1}$ and $k_{3}$ were lower than the analogous experiment with no $\mathrm{KOH}$ (entry 1).

The effect of A336 in the present reaction seems to be similar to that observed for 1,3,5-TCB (Fig. 3) and the working amount of $0.2 \mathrm{mmol}$ of A336 corresponds to the 
saturation of the rate dependences on the amount of A336. In fact, a threefold excess of A336 (entry 8, Table 3) does not affect the reaction rates. A twofold increase in the amount of Raney-Ni instead produces the twofold increase in the reaction rates (entry 7 , Table 3 ).

\section{CONCLUSIONS}

In the reductions of 1,3,5-TCB and $p$-chloroacetophenone over Raney-Ni with hydrogen under multiphase conditions it was found that the components constituting the multiphase environment, namely the PT agent and the aqueous base, have great influence over the reaction rate and selectivity and are even indispensable to the hydrodechlorination of 1,3,5-TCB.

The dependences of dechlorination rates of 1,3,5-TCB on the concentration of the aqueous base manifest a maximum reached at moderate base concentrations $(3 \% \mathrm{KOH}$ or $10 \% \mathrm{~K}_{2} \mathrm{CO}_{3}$ ). Solid bases appear to be less efficient, while the use of aqueous carbonates and bicarbonates results in higher dechlorination rates. In the reduction of carbonyl group in $p$-chloroacetophenone the presence of A336 and of base seem not to be critical, though the presence of base suppresses the successive reduction of hydroxyl.

The dechlorination rates of 1,3,5-TCB depend linearly on the concentration of A336 at low A336 concentration and reach a plateau at $0.2 \mathrm{mmol}$ of $\mathrm{A} 336$. The cocatalytic effect of A336 seems to be connected to its adsorption on the catalyst surface, where the only A336 species active are the ones that are in direct contact with the Raney-Ni surface.

The research on the mechanistic features and applications of the Raney-Ni- catalyzed hydrogenation/ hydrogenolysis under multiphase conditions will be further extended in the future studies.

\section{ACKNOWLEDGMENTS}

This work was partially funded by INTAS 2000-00710 and NATO EST.CLG.977159 grants and by the Interuniversity Consortium INCA (www.unive.it/inca/). Sergei Zinovyev also thanks the INTAS fellowship YSF-00-138. We also gratefully acknowledge Engelhard for the gift of the Raney-Ni catalyst.

\section{REFERENCES}

1. Marques, C. A., Selva, M., and Tundo, P., J. Chem. Soc. Perkin Trans. 1529 (1993).

2. Marques, C. A., Selva, M., and Tundo, P., J. Org. Chem. 58, 5256 (1993).

3. Marques, C. A., Rogozhnikova, O., Selva, M., and Tundo, P., J. Mol. Catal. A 96, 301 (1995).

4. Marques, C. A., Selva, M., and Tundo, P., J. Org. Chem. 59, 3830 (1994).

5. Marques, C. A., Selva, M., and Tundo, P., J. Org. Chem. 60, 2430 (1995).

6. Selva, M., Tundo, P., and Perosa, A., J. Org. Chem. 63, 3266 (1998).

7. Perosa, A., Selva, M., and Tundo, P., J. Org. Chem. 64, 3934 (1999).

8. Tundo, P., Zinovyev, S., and Perosa, A., J. Catal. 196, 330 (2000).

9. Hoke, J. B., Gramiccioni, G. A., and Balko, N. L., Appl. Catal. B 1, 285 (1992); Pasett, P., and Gentile, F., J. Org. Chem. 60, 1351 (1995).

10. Bomben, A., Marques, C. A., Selva, M., and Tundo, P., Synthesis 1109 (1996).

11. Perosa, A., Tundo, P., and Selva, M., J. Mol. Catal. A 180, 169 (2002).

12. Tundo, P., Perosa, A., Selva, M., and Zinovyev, S. S., Appl. Catal. B 32, L1 (2001).

13. Schmid, R., and Sapunov, V. N., "Non-Formal Kinetics." Verlag Chemie GmbH, Weinheim, 1982.

14. Beranek, L., Adv. Catal. 24, 1 (1975).

15. Landini, D., Maia, A., Montanari, F., and Pirisi, F. M., unpublished results.

16. Demlow, E. V., and Demlow, S. S., "Phase-Transfer Catalysis," 3rd ed. Verlag Chemie GmbH, Weinheim, 1983.

17. Yufit, S. S., "Mekhanizm Mezhfaznogo Kataliza." Nauka, Moscow, 1984 (in Russian).

18. Engelhard assistance service, tel. +31-(0)30-666 9555 . 